\title{
The Flexion Properties of a 3D Printed Structure Designed using Auxetic Mechanical Metamaterials
}

\author{
Nicole Seman \\ Academy of Science \\ Academies of Loudoun \\ Leesburg, United States
}

\author{
Sundaram Thirukkurungudi \\ Academy of Science \\ Academies of Loudoun \\ Leesburg, United States
}

\begin{abstract}
D printing technologies can be used to create simple, yet effective gripping mechanisms. By implementing a bow-tie shaped mechanical metamaterial in a dexterous model, structures can be actuated without motors. This experiment investigates the flexion angles of joints in a 3D printed grasping device with an integrated mechanical metamaterial design. A mechanical metamaterial has properties defined by its structure as opposed to composition. If mechanical metamaterials are applied to a gripping device, then actuation will be achieved by applying a pressure to the structure surface. To construct such a joint, appendages were to be printed using NinjaFlex. Printing, as opposed to using a mold makes modelling simpler and decreases the number of materials needed.
\end{abstract}

Force was then applied to the model using a Mark 10 machine and the flexion angles of the model were measured using a protractor. Using the flexion angles calculated at varying forces, inverse exponential regressions were fitted to the data taken at each pivot distance for each of 4 designed structures. ANOVA tests determined that a structure with a tilted bowtie pattern (4) bent significantly more than other structures.

To determine if silicone would be a better material for the structures, a K8200 Fused Deposition Modeling (FDM) printer was equipped with a paste extrusion system. Such a system utilizes a syringe of material as opposed to heated filament in the deposition process. During this process, multiple silicone prints were done to find the optimal settings for this system.

Keywords - mechanical metamaterials, 3D printing, paste extrusion, flexion, bowtie design

\section{INTRODUCTION}

With an increase in automated mechanisms, robotic grippers are becoming prevalent; utilized in machines working in dangerous situations, prosthetics, and machining automation. The complexity of such grasping devices has increased over the years: advancing from simple jointed systems to the intricate dexterous machines which are used today. Extremely nimble grippers consist of many joints as well as complex mechanical and technological systems. However, in many applications where such a device would be beneficial, funding is not substantial, these devices are difficult to produce, and few are made. Furthermore, the excessive weight of such a machine may be detrimental in many environments where a gripper would be required. An alternative method of designing a gripper is being investigated in the following study. This design uses a mechanical metamaterial design in the joints of a modelled grasping device. Overall, this integration aims to decrease the complexity in manufacturing gripping devices.
The proposed investigation intends to determine which structure, designed using mechanical metamaterials, has the optimal flexion trends for use in an efficient grasping mechanism. It was hypothesized that if a bow-tie shaped mechanical metamaterial is implemented in a mechanism, then actuation will be achieved by applying a pressure to the metamaterial surface. Additionally, if the force applied to the structure is altered, then as force is increased, flexion angles will increase until reaching a limiting point. The design of the printed structure will be changed, and the flexion angle of the structures at different points will be measured. This information is significant because it will be used to determine the optimal mechanical metamaterial design for flexion to be implemented into a grasping mechanism.

\section{BACKGROUND}

Metamaterials are commonly defined as manmade compounds which have physical properties determined by their macro or micro structure rather than the specific substance they are composed of. They exhibit properties not found naturally in nature. Although primarily known for having a negative refractive index, which gives objects the ability to appear invisible, other metamaterials have been engineered to have mechanical properties not occurring naturally. Specifically, an auxetic mechanical metamaterial has been engineered. These metamaterials have been constructed using 3D printing with a patterned structure of rigid sections connected by flexible pivots. By applying a pressure to the structure, the patterned sections can be twisted to transform the system to an alternate configuration. [6].

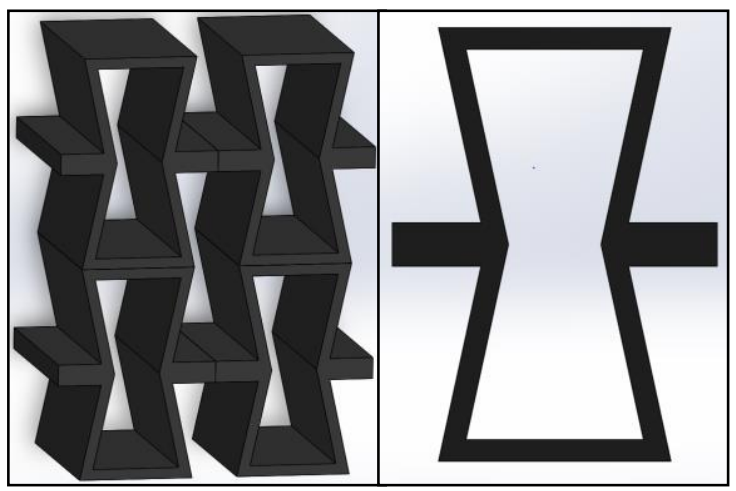

Fig. 1. Solidworks model of bowtie pattern utilized in the design of each structure. 


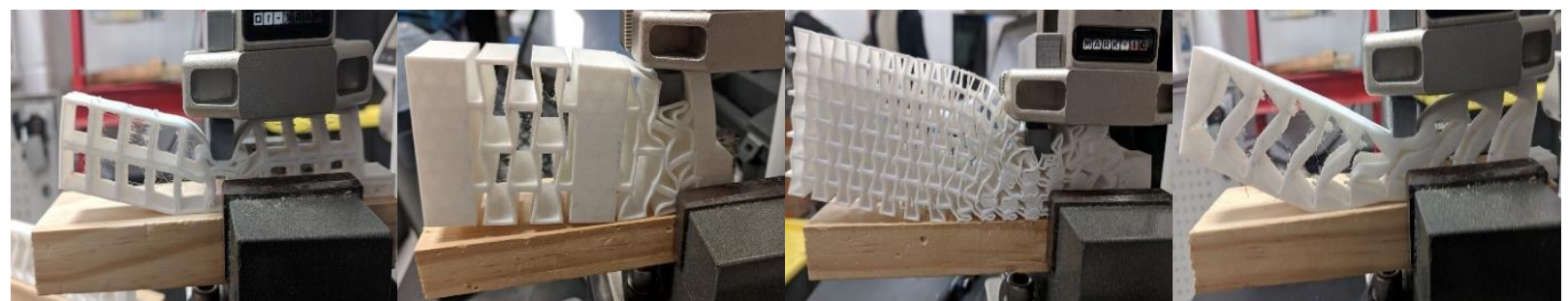

Fig. 3. Flexion angle testing done on tensile strength machine. Structures $1-4$ are shown from left to right.

\section{MATERIALS AND METHODS}

NinjaFlex, a flexible TPU filament, was determined to be successful for printing the structures with mechanical metamaterials in conducting experiments on the effectiveness of structure shape. 4 structures were then designed, each with varying characteristics thought to change the flexion trends. Structure 1 was a control design, with a cutout pattern of $0.5 \mathrm{in}$ $\mathrm{x} 0.5$ in squares, 2 rows high. Structure 2 had a 2-row high, basic bowtie pattern, with shapes 1 in tall. The third structure had smaller bowtie instances, with 4 rows of bowties $0.5 \mathrm{in}$ tall. A final structure utilized a slightly larger bowtie pattern, with the instances tilted at a 45-degree angle. These designs can be seen further in Figure 2. An anchor was designed at the end of each structure for attachment to a base piece. The structures were formatted for printing using Cura. An extruder temperature of 240 degrees Celsius and a bed temperature of 40 degrees Celsius were set.

The structures were then attached to a cut piece of lumber, each approximately $0.5 \times 0.75 \times$ in in size. The anchors of the structures were superglued on the end of the wood pieces and the apparatuses were given an hour minimum to cure. To prepare for flexion testing, an anvil shaped piece was 3D printed using PLA, which was to be used for applying a force to the structure.

The wooden bases were then clamped in to the Mark 10 ESM Compression Test Stand. The printed anvil piece was secured to the force application portion of the machine. 3 different points along the structure were selected for force testing in order to get a comprehensive and optimal understanding of the flexion trends in the structure. For each point, the structure was positioned under the anvil load at a certain location, which was then measured by taking the distance from the edge of the pivot to the end of the structure opposite the anchor. This was described as the pivot distance. After the piece was secured in the machine, the load was lowered until just slightly touching the structure and the first force measurement was taken, with a corresponding offset distance. The offset distance was defined as the distance from the top surface of the wood piece to the bottom edge of the structure furthest from the anchor. Following this initial measurement, 5 additional measurements were taken by lowering the load and stopping it at certain intervals where motion had been observed. The measured force on the structure was then measured in LbF from the force gauge and the corresponding offset distance was calculated. This set of procedures was repeated for the specific pivot distances. As previously stated, measurements were conducted at 3 points along the structure and done for each structure. Following these measurements, flexion angles were determined (1).

$$
\text { flexion }\left(^{\circ}\right)=\tan ^{-1}\left(\frac{\text { offset }(\mathrm{mm})}{\text { pivot }(\mathrm{mm})}\right) *\left(\frac{180}{\pi}\right)
$$

\section{NINJAFLEX STRUCTURES}

In experimentation conducted utilizing NinjaFlex, flexion trendlines were constructed using LoggerPro, comparing the flexion angles vs. the applied force for each pivot distance of each structure. Inverse exponential regressions were fitted to the data, with flexion angle being a function of applied force, $F_{a}(2)$.

$$
\text { flexion }^{\circ}=A\left(1-e^{-C\left(F_{a}\right)}\right)
$$

The "A" coefficient was determined to represent the maximum flexion in the structure and the " $\mathrm{C}$ " coefficient was determined to represent the rate of change in flexion trends. Larger " $A$ " values correlated to a higher maximum bending and larger " $C$ " values to a more asymptotic relationship. This regression pattern was selected because of the asymptotes produced, which could be used to represent the leveling out of flexion angles with an increase in force applied. These trends can be viewed in Figures 13-16 of the appendix.

In Structure 1, the 3 pivot distances yielded similar trends of increasing flexion then asymptotic behavior; with the graphs clearly distinguishable. A pivot distance of $67 \mathrm{~mm}$ was determined to be optimal, with an "A" coefficient of 15.54 and a "C" coefficient of 0.0806 . Structure 2 had more variable flexion patterns. Specifically, the trendline for a $99 \mathrm{~mm}$ pivot distance did not reach a visible asymptote as rapidly as the other graphs and although lower than the $44 \mathrm{~mm}$ pivot initially, passed it as the $44 \mathrm{~mm}$ pivot graph trendline reached an asymptote. This equation had a " $\mathrm{A}$ " and " $\mathrm{C}$ " coefficients of 6.186 and 0.04803 , respectively. The flexion trends for Figure 3 were highly variable and best fit equations were only able to be fitted to 2 of the pivot distances. These regressions did not reach visible asymptote and had extremely high standard errors. Overall, a $58 \mathrm{~mm}$ pivot distance was determined to have the best flexion measurements in this collection of data, with an " $A$ " value of 23.14 and a " $C$ " value of 0.005558 . Finally, Figure 4 revealed distinct inverse exponential relationships for all pivot distances. Rapid increases in flexion occurred initially, with clear asymptotes reached. The optimal flexion occurred with a pivot distance of $127 \mathrm{~mm}$ and provided an " $\mathrm{A}$ " coefficient of 23.29 and a "C" coefficient of 0.2653 . This structure had the highest calculated flexion angle at any point, with a value of approximately 23 degrees. 


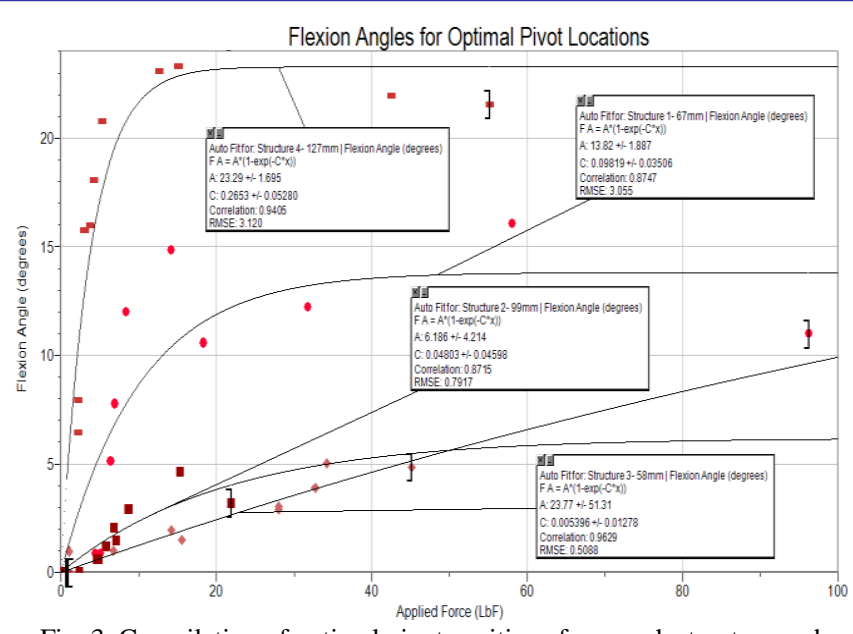

Fig. 3. Compilation of optimal pivot positions from each structure and their respective trendlines.

A visual analysis was done to determine these optimal pivot distances within each structure. Although regressions did not fit the data exactly, correlation coefficients were primarily above 0.8, with two being 0.52 and 073 in Structure 2 . Standard errors for the "A" and "C" coefficients were calculated to account for the inaccuracies in trendlines.

A cross structure comparison was then conducted on regressions with the optimal pivot distances (Figure 5). 2 oneway ANOVA test were used for comparison of "A" and " $C$ " coefficients. A parametric test was conducted because the data was derived from mean and standard errors as opposed to the raw data needed for a nonparametric study. Structure 3 was omitted from these comparisons because of extremely high standard error in the inverse exponential coefficients that invalidated conclusions which could otherwise be drawn.

P-values from the ANOVA test comparing both coefficients determined that there was an overall statistically significant difference in the flexion patterns of the 3 structures. However, both the "A" and "C" coefficients compared between Structures 1 and 2 were not significant, with p-values greater than 0.05 , the significance level. All comparisons to Structure 4 were significant, the comparisons with Structure 1 yielded pvalues less than 0.05 and the comparisons with Structure 2, less than 0.001. These latter comparisons were considered extremely significant. This data can be supported by confidence intervals constructed for each coefficient. None of the values overlapped, and Structure 4 had the highest interval. This can be supported by the exact values in Figures 6 and 7 .

\section{PASTE EXTRUSION METHODS}

A Velleman k8200 Fused Deposition Modelling (FDM) printer was constructed and the printer was modified to have a paste extrusion system. This system works by extruding the material from a syringe as compared to heating a filament.
Prior to successful printing, the printer's controller had to be reflashed. Arduino software was downloaded and was first used to disable the heaters on both the bed and extruder by setting the minimum temperatures to 0 . This was necessary for extrusion to be possible without the thermistors detecting a temperature capable of melting PLA filament. After the extrusion motor, it was observed that extrusion commands resulting in retraction motions and vice versa.

Printing was then conducted using a $60 \mathrm{~mL}$ syringe with a blunt, 16G needle tip and DAP $100 \%$ Silicone. 4 trials using silicone were attempted, with various printer settings being adjusted each time. This was adjusted by accessing the Slic3r settings through Repetier-Host. These settings most prevalently included the addition of a Z-lift and after layer gcode. The $Z$-lift moved the $Z$ axis up a specified distance before all nonconsecutive print moves. This was intended to decrease the drag of silicone by the nozzle. An after-layer gcode addition was programmed to move the printing nozzle up then $50 \mathrm{~mm}$ to the left, situated near the edge of the print bed. The nozzle paused there for 20 minutes, to allow the silicone time to harden before a new layer was started. Other settings adjusted included print speed, addition of a UV light, securing the paste-extrusion assembly, extrusion multiplier, nozzle diameter, filament diameter, retraction length, and layer height. The adjustment of these settings was intended to improve print quality. The results of these adjustments can be seen in the qualitative analysis of the 4 trials.

\section{RESULTS OF SILICONE EXTRUSION}

A qualitative analysis of the first trial revealed that silicone needed more time to harden before successive layers were started and an incorrectly set nozzle diameter. The silicone drag was very high in this attempt. For trial 2, the filament diameter was increased to slow down extrusion and the print speed was slowed down. In the third trial, a $5 \mathrm{~mm}$ Z-lift was added and retraction length was increased to $10 \mathrm{~mm}$. Furthermore, an after-layer g-code pause was added. This allowed the trial to print an addition 5 layers, as compared to the previous trials. With the additional layers, there was an increased amount of silicone excess. This was remedied by a fourth trial, where retraction was increased to $300 \mathrm{~mm}$ and the Z-lift to $10 \mathrm{~mm}$. This trial also resulted in the printing of 6 layers and was of the best quality. Overall, the print trials only reached a maximum of 6 layers; approximately 13\% completion, however, print quality increased with each of the 4 trials conducted and the final combination of settings could be utilized to successfully extrude silicone. Silicone printing could be used as an effective alternative to mold-based manufacturing.

It was decided that the printing of silicone was too tedious to be effective in a proof-of-concept study. Additionally, it was observed from the trials that silicone was extremely flexible and could impact the ability of the flexion trends to be accurately compared between the different structures printed. 


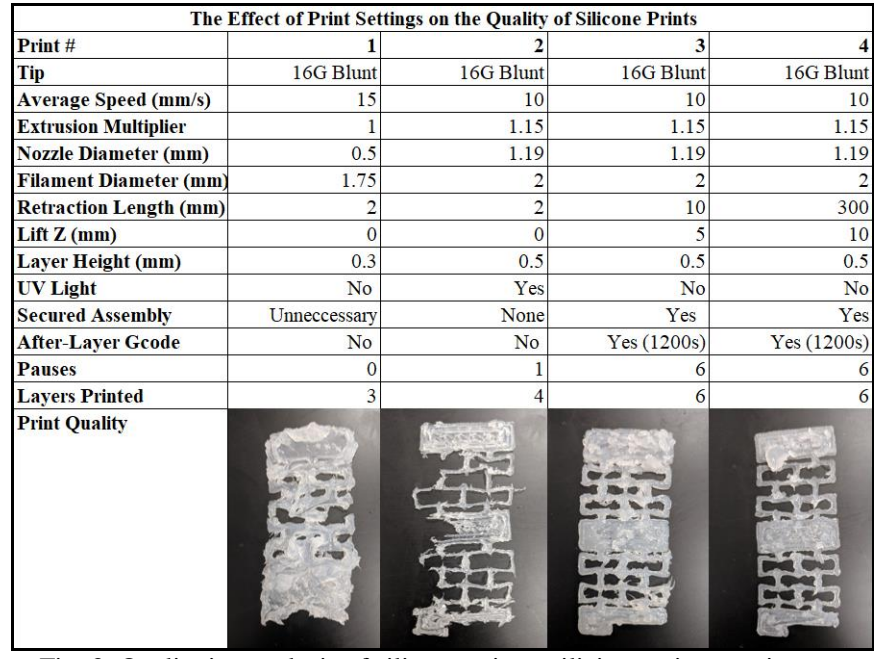

Fig. 3. Qualitative analysis of silicone prints utilizing various settings, adjusted on the printer and in the slicer.

\section{LIMITATIONS AND FUTURE WORK}

Possible limitations throughout experimentation include imperfections in print quality of the NinjaFlex structures and disparities between the structures. In an optimal investigation, more structures would have been printed, with just one variable changed between each structure. This would give a more accurate analysis of the effectiveness of each aspect of the mechanical metamaterial design. Additionally, the structures were not centered on the Mark 10 device and this could have caused inaccuracies in the offset distance measurements. A final limitation could be the inverse exponential equations fitted to the data, as this is possibly not the most accurate portrayal of the data.

In future continuations of this study, a first improvement would be to account for the aforementioned limitations. Second, the effect of different mechanical metamaterial patterns will be investigated, using designs with a variety of shapes. After this is tested, the effect of angling the patterns, such as what was done in Structure 4 should be investigated; as this had a drastic effect on the flexion results.
Finally, the optimal structures should be designed to be implemented and tested in a grasping mechanism to determine the feasibility of a real-life application of the research and the increased effectiveness of a grasper using this concept. When the structure design is finalized, comparisons can be done on the effectiveness of the structures when different materials are used; such as silicone, NinjaFlex, and other flexible options.

\section{CONCLUSION}

It can be concluded that the structures, from least to most effective, were $2,1,4$. A control, Structure 1, bent marginally more than Structure 2, which utilized vertical bowtie pattern. Structure 4 , with a tilted bowtie pattern, bent significantly more than other structures. The hypotheses: if a bow-tie shaped mechanical metamaterial is implemented in a mechanism, then actuation will be achieved by applying a pressure to the metamaterial surface, and if the force applied to the structure is altered, then as force is increased, flexion angles will increase until reaching a limiting point, were proven valid. However, it is important to note that the deformation was most effective when a tilted bowtie design was implemented.

\section{REFERENCES}

[1] Belter, J.T., Segil, J.L., Dollar, A.M., \& Weir, R.F. (2013). Mechanica design and performance specifications of anthropomorphic prosthetic hands: A review. Journal of Rehabilitation Research and Development (JRRD), 50(5), 599.-618. doi: 10.1682/JRRD.2011.10.0188

[2] Deimel, R, Brock, O (2015). A Novel Type of Compliant and Underactuated Robotic Hand for Dexterous Grasping. The International Journal of Robotics Research, 35(1), 161.-185. doi: $10.1177 / 0278364915592961$

[3] Feix, T., Romero, J., Schmiedmayer, HB., Dollar, A.M., \& Kragic, D. (2015). The GRASP Taxonomy of Human Grasp Types. IEEE Transactions on Human-Machine Systems, 46(1), 66.-77. doi 10.1109/THMS.2015.2470657

[4] Kim, H., Han, MW., Song, SH., \& Ahn, SH. (2016). Soft morphing hand driven by SMA tendon wire. Elsevier Composites Part B, 138 . 148. doi: 10.1016/j.compositesb.2016.09.004

[5] Mark, A.G., Palagi, S., Qiu, T., \& Fischer, P. (2016). Auxetic Metamaterial Simplifies Soft Robot Design. 2016 IEEE International Conference on Robotics and Automation, 4951.-4956. doi: 10.1109/ICRA.2016.7487701

[6] Rocklin, Z., Zhou, S., Sun, K., \& Mao, X. (2015). Transformable topological mechanical metamaterials. Nature Communications, 8 , 14201. doi: $10.1038 /$ ncomms 14201

[7] Rus, D., Tolley, M. (2015). Design, fabrication and control of soft robots. Nature, 521, 467.-475. doi: 10.1038/nature14543Science, 1989 